\title{
The model of seal mechanism for magnetic fluid and related experimental study
}

\author{
Sihai Zhao*, Qiang Sheng**, Sen Lin***, Fan Zhang****, Lingyu Jiao***** \\ *China University of Mining \& Technology (Beijing), Beijing 100083, China, E-mail: zsh@cumtb.edu.cn \\ **China University of Mining \& Technology (Beijing), Beijing 100083, China, E-mail: 15201308273@163.com \\ ***China University of Mining \& Technology (Beijing), Beijing 100083, China, E-mail: linsen199006@163.com \\ ****China University of Mining \& Technology (Beijing), Beijing 100083, China, E-mail: 1043769633@qq.com \\ *****China University of Mining \& Technology (Beijing), Beijing 100083, China, E-mail: $491165440 @ q q . c o m$ \\ cross $^{\text {ref }}$ http://dx.doi.org/10.5755/j01.mech.22.4.16156
}

\section{Introduction}

Magnetic liquid as a kind of new functional material, magnetic fluid sealing is the most important application. At present, the rotating magnetic liquid seal is comparatively mature technology, more mature products have been designed at home and abroad, widely used in all kinds of vacuum equipment, Such as swing machine, coating machine and single crystal silicon furnace, etc $[1,2]$. But reciprocating shaft seal of magnetic liquid in the theory, experimental research and practical application are not mature, the mainly problems include: 1) How to accurately draw the quantitative relationship between the parameters of movement of the reciprocating shaft and parameters of seal structure with the compression ability? 2) How to analyze the flow mechanism of magnetic liquid inside sealing gap? 3) How to accurately draw the motion state of magnetic fluid when the reciprocating shaft moves [3].

This paper obtains from the magnetorheological effect of magnetic fluid, the yield stress formula of magnetic liquid was deduced, based on the the yield stress formula of magnetic liquid, The mechanism of magnetic fluid seal is analyzed, the pressure formula of magnetic fluid seal is Concluded, and then the experimental investigation was conducted.

\section{Magnetic liquid seal}

Magnetic fluid or magneto-fluid is a kind of colloidal solution with ultrafine ferromagnetic particles with grain diameter of $10 \mathrm{~nm}$ and interfacial agent dispersed steadily in liquid. It doesn't agglomerate or deposit even under the effect of common centrifugal force and magnetic field and appears as a kind of fluid with magnetism. As a new kind of liquid functional material, magneto-fluid is widely applied in many fields, among which seal is the most typical one. At present, the application of magneto-fluid in vacuum and gas seal has been at practical stage $[4,5]$.

Magnetic fluid seal of rotation shaft is a kind of non-contact seal, with a closed magnetic circuit consisted by a ring permanent magnet, magnetic pole shoes and a magnetic rotation shaft. By taking advantage of the magnetic energy of permanent magnet, non-uniform magnetic field with strong-weak distribution is generated in the gap between the rotation shaft and the teeth of pole shoes. The magnetic fluid is drawn tightly and an O-type ring of magnetic liquid is formed with the gap blocked off, therefore, the purpose of seal is achieved.
Essentially, magnetofluid or magnetic liquid is a kind of magnetorheological fluids (MRF), only its yield stress is lower than conventional magneto rheological fluid [5].

MRFs refer to materials which experience rapid changes in their rheological behaviors when placed in external magnetic field. Their basic characteristic is that they experience a reversible change from free-pouring liquid state into semisolid state with controllable yield strength after exposure to strong magnetic field. The effect of magnetic field on characteristics like viscosity, plasticity and viscoelasticity is named as magnetic rheology effect $[6,7]$.

The seal function of magnetic liquid can be explained by magnetic rheology effect. A relatively strong permanent magnet is placed at positions where seal is required. When magnetic liquid flows through, chain structures are formed along the magnetic force line under the effect of magnetic field [8]. Then, the fluid is constrained and cannot flow until the pressure difference between internal surface and external surface is higher than that produced by the yield stress of MRF. Therefore, seal is achieved.

\section{Magnetic agglomeration of MRF caused by external magnetic field}

With surfactant added, MRF shows better dispersion stability without external magnetic field and the particles reject with each other.

When MRF is in external magnetic field, according to the theory of DLVO and its extension in the study of magnetic agglomeration, at the same time considering the solvation membranes rejection potential energy, the total interaction energy $U_{T}$ between the ferromagnetic particles in suspension is [9]:

$$
U_{T}=U_{A}+U_{E L}+U_{H R}+U_{M P},
$$

where $U_{A}$ is the Van Der Waals potential energy; $U_{E L}$ is the double electrode layer potential energy; $U_{H R}$ is the solvation membranes rejection potential energy; $U_{M P}$ is the magnetic interaction energy between ferromagnetic particles.

$$
U_{M P}=\frac{1}{\mu_{0} a^{3}}\left[m_{1} m_{2}-3\left(m_{1} a\right)\left(m_{2} a\right) a^{-2}\right],
$$

where $\mu_{0}$ is vacuum permeability; $m_{1}$ and $m_{2}$ are the magnetic moments of Particle 1 and Particle 2 respectively, while $a$ is the center distance between two particles. 


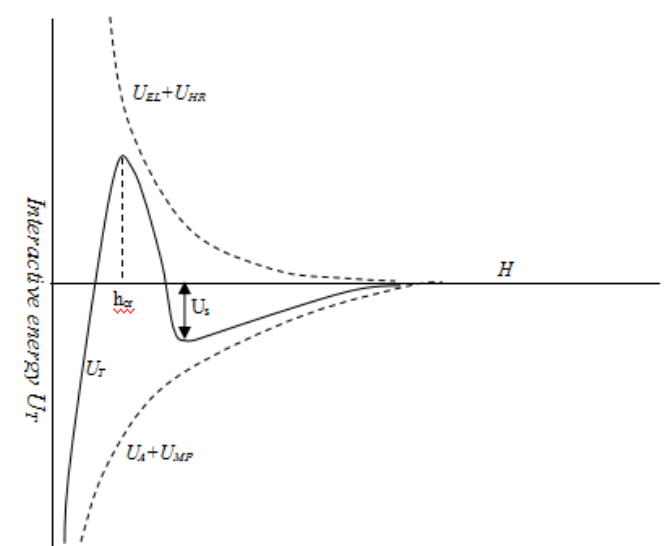

Fig. 1 The general potential energy of interaction between particles (DLVO)

In Fig. 1, it is showed that $U_{E L}$ and $U_{H R}$ is repulsive interaction, and $U_{A}$ and $U_{M P}$ is attractive interaction. So $U_{E L}$ and $U_{H R}$ at the horizontal line above, $U_{A}$ and $U_{M P}$ at below of the horizontal line. The horizontal line show that the the total interaction energy $U_{T}$ varies with the distance between particles.

In comparison with the Van Der Waals potential energy, the double electrode layer potential energy and the solvation membranes rejection potential energy, the magnetic force is the greatest of all. When the external magnetic field applied, magnetic attraction becomes the most important of all, and the general applied force between particles is attraction. Therefore, particles begin to agglomerate. The phenomenon that particles under the effect of external magnetic field agglomerate mainly depending on magnetic attraction is named as magnetic agglomeration. In external magnetic field, ferromagnetic particles are in the condition of chain agglomeration state, and the length direction of chains in magnetic agglomeration groups always keeps the same with that of the external magnetic field basically.

When MRF is under the effect of external magnetic field, an overall interattraction phenomenon is shown among ferromagnetic particles in companion with the occurrence of magnetic agglomeration. Chain-like flocculation comes into being, and then network structures are formed in the entire liquid. Parts of continuous phases are wrapped, resulting in the increase of effective phase volume, i.e. the increase of viscosity. When the flocculated MRF is sheared, the flocculation constituents rotate, deform and even crack when the applied force is strong enough.

With the magnetic induction intensity of external magnetic field increasing, the linear extent of flocculation grows obviously by developing from dimers or triplets into concatemers with the occurrence of branched chains.

Therefore, under the effect of external magnetic field, the magnetic agglomeration of MRF is the main cause of the occurrence of chain-like structure in MRF magnetic particles [10].

\section{Yield stress of MRF in external magnetic field}

Without the influence of external magnetic field, MRF shows no characteristic related to plastic fluid and is supposed to have favorable dispersion stability, i.e. repulsive interaction is dominant among its internal ferromagnetic particles. However, when external magnetic field is applied, magnetic interaction among ferromagnetic particles becomes important of all, resulting in the inter-attraction among particles which leads to magnetic agglomeration. The product of magnetic agglomeration is a kind of chainlike flocculation, which forms network structure with external magnetic field increasing. When MRF is under shear stress, flocculation constituents rotate, deform and even crack when the force applied is strong enough. This is the main reason of MRF acting as plastic fluid under the external magnetic field applied, and the structural strength of chain-like flocculation structures in MRF determines the yield stress of MRF.

Based on previous discussions, it can be concluded that the yield stress of MRF is depended on the structural strength of flocculation structures formed during magnetic agglomeration. According to experimental and theoretical analysis, the liner extent of chain-like flocculation constituents grows obviously with the increase of external magnetic field intensity, and concatemers come into being. Thus, the structural strength of magnetic agglomerated flocculation constituent is improved, resulting in the increase of MRF yield stress.

Assuming that all particles have a same diameter, then under the effect of external magnetic field, the magnetic attraction force between ferromagnetic particles is [11]:

$$
F_{m}=3.898 D M^{2} R^{2}(1-\varepsilon)^{\frac{4}{3}},
$$

where $D$ is the demagnetization factor of particle and vacuum, and it is a constant for a specific particle. $R$ is the diameter of particles, $\varepsilon$ is the void ratio of MRF and generally $\varepsilon=1-C$, where $C$ is the volume concentration of particles in MRF. $M$ is the magnetization intensity of particles.

Since the magnetic attraction force is greater than other surface force with the existence of external magnetic field, the effect of other surface force is no considered here.

Fig. 2 show that two kinds of work pattern for MRF, one is external force mode, show as 2(a), it is often work in static seal. the other is shear mode, show as 2(b), it is work in rotary seal. General magnetic fluid seal is the superposition of this two kinds mode. After ferromagnetic particles forming chain-like flocculation due to magnetic agglomeration in external magnetic field, the attraction between particles is $F_{m}$. When the chain-like flocculation constituent is bended by external force, component forces of $F_{m}$ along $X$ direction and $Y$ direction are produced as $F_{m x}$ and $F_{m y}$, respectively. When the external force is smaller than $F_{m x}$, the base fluid doesn't flow under the constraint of chain-like flocculation. When the external force is greater than $F_{m x}$, particles are separated from their attachment points of magnetic attraction. The chain-like flocculation constituent moves or even crack, and the base fluid becomes mobile accordingly. Assuming that there are $n$ flocculation constituent chains per unit area, and the macro performance of $F_{m x}$ of all the magnetic agglomerated chain-like flocculation constituents within the unit area is the dynamic yield stress, then the force required to break or move flocculation chains is the yield stress [11].

$$
\tau_{y}=n F_{m x}=3.898 n D R^{2}(1-\varepsilon)^{4 / 3} M^{2} \sin \alpha,
$$

where $\alpha$ is the maximum deviation angle of chain-like flocculation constituent under the effect of external force. 
It can be learnt from Eq. 4 that the yield stress is proportional with the magnetization intensity of particles. Magnetization intensity $M$ is equal to the product of mag-

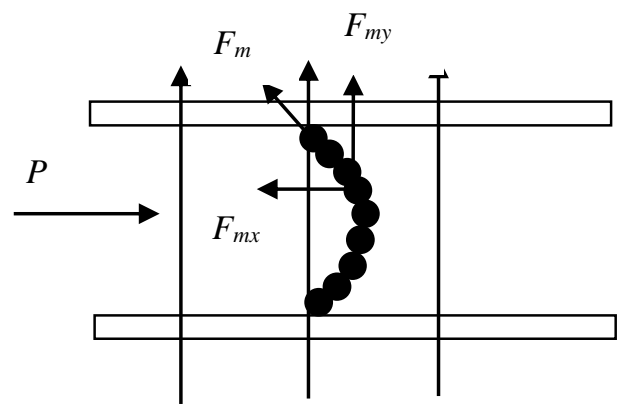

a netic susceptibility $\chi_{m}$ and magnetic field intensity $H$, i.e. the yield stress is proportional with the square of external magnetic field intensity.

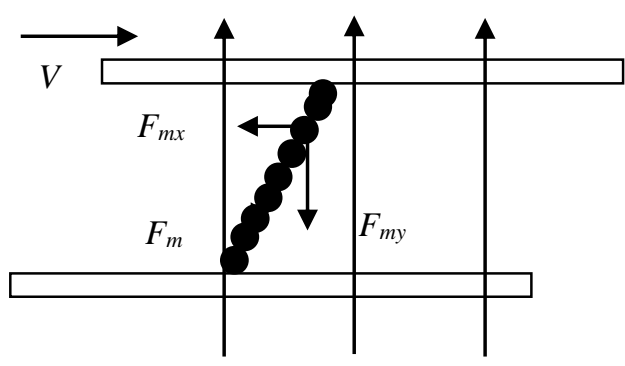

b

Fig. 2 The distortion of chain-like flocculation under external force

Previous experiments and studies reveal that before reaching saturation the yield stress of MRF increases with magnetic field intensity $H$ or magnetic induction intensity $B$. Ginder further found via finite element simulation that only at relatively low magnetic field intensity sections, the yield stress was in direct proportion with $H^{2}$. For greater magnetic field intensity, the polarization area of each particle became locally saturated and the corresponding yield stress was in direct proportion with $H^{3 / 2}$. When the magnetic field intensity was great enough to reach total saturation, all the particles could be regarded as doublets, and the yield stress had nothing to do with the magnetic field, but had a direct proportion with $M_{s}^{2}$ [9].

Based on Eq. 4 and the relationship between magnetization intensity and magnetic field intensity explained by electromagnetism, the previously mentioned relationship among yield stress, magnetic field intensity $H$ and saturation magnetization intensity $M_{s}$ can be successfully explained, which also proves the validity of chain-like flocculation theory of magnetism agglomeration.

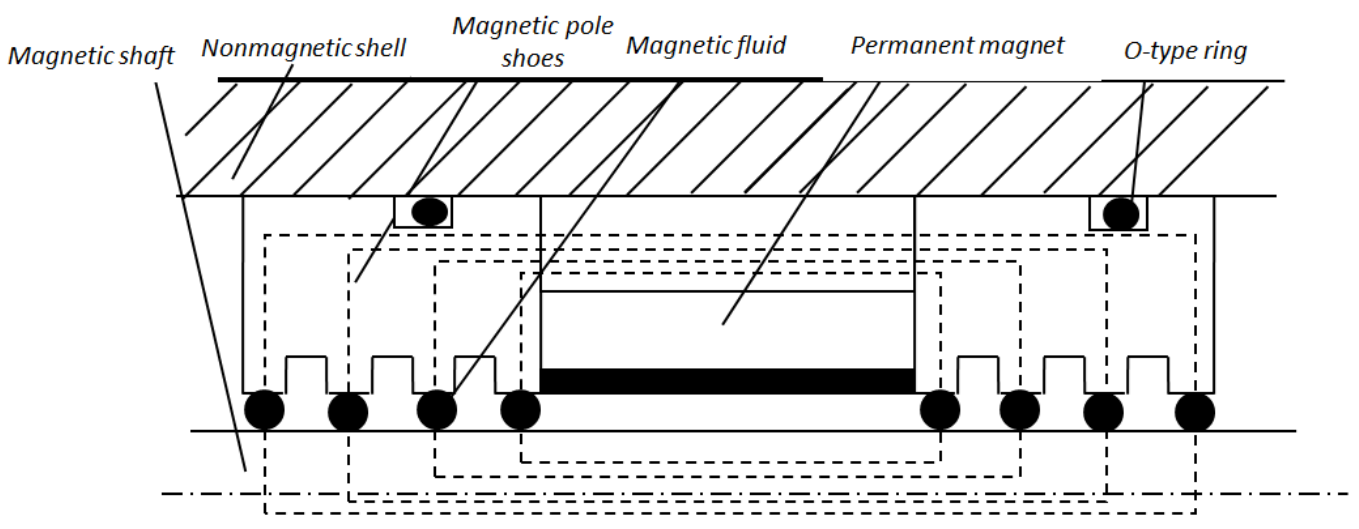

Fig. 3 The structure of magnetic fluid seal

\section{Principle and structure of magnetic fluid seal}

MRF is a kind of plastic fluid, which is also known as Bingham fluid. In external magnetic field, the constitutive equation of Bingham fluid can be adopted for MRF [6]:

$$
\tau=\tau_{0}+\eta \dot{s}
$$

where $\tau$ is the shear stress, $\tau_{y}$ is the yield stress, $\eta$ is the stiffness coefficient, $\dot{s}$ is the shear rate.

The yield stress of MRF, which indicates its solidification strength, is the major parameter of this kind of material.

The principle of typical magnetic fluid seal is shown as Fig. 3. Considering that magnetic fluid is a kind of MRF, the seal process and corresponding principle can be explained using magnetic rheology effect [12].

As mentioned earlier, under the large action of external magnetic field, the magnetic particles can form chain structure, the viscosity of magnetic fluid has been changed. Magnetic fluid is consisted by nano-sized ferromagnetic particles with low saturation magnetization intensity, which is set as $M_{s}$. Assuming that under the effect of permanent magnet, magnetic fluid has reached its saturation magnetization intensity, then the yield stress of magnetic fluid is proportional with the square of saturation magnetization intensity $M_{s}[13]$.

As shown in Fig. 3, when the distance between the teeth of magnetic pole shoes and magnetic shaft (black dot shown in Fig. 3, expressing magnetic fluid) is short during magnetic fluid seal, the magnetic induction intensity is relatively high. Due to the magnetic rheology effect, chain-like flocculation structure is formed along magnetic force line between the teeth of magnetic pole shoes and magnetic shaft. All liquid is constricted around by this structure and unable to flow any more. Therefore, the seal of both internal and external cavities is accomplished.

In magnetic fluid seal clearance, there is a larger 
magnetic field intensity $\mathrm{H}$, magnetic particles in gap will form the same chain structure. when there is a differential pressure, magnetic liquid will produce bending chain, as shown in Fig. 2, a. Due to the attractive force between magnetic particles, the force in horizontal direction to create a component force to resist the pressure $P$. When the top plate moves, the chain also moves, the chain generates tilt, as shown in Fig. 2, b, Horizontal direction to produce a restoring force, this two kinds of force is generated by the yield stress.

Under the effect of magnetic field, MRF is a kind of plastic fluid and confirms to Bingham model. Similarly, magnetic liquid also conforms to Bingham model [14]. According to Bingham model, fluid can flow only when $P$ is higher than $\Delta P \tau$.

$$
\Delta P_{\tau}=\frac{3 L \tau_{0}}{b}
$$

where $b$ is the gap between magnetic shaft and the teeth of magnetic pole shoes, $L$ is the length of each tooth and $\tau_{0}$ is the yield stress of magnetic fluid which can be determined in experiments.

For magnetic liquid between the teeth of magnetic pole shoes and magnetic shaft, Fig. 4 is the schematic of magnetic fluid under external force [15].

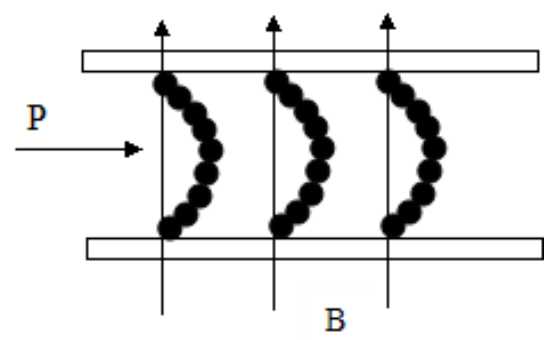

Fig. 4 The sketch map of magnetic fluid under an applied force

The total pressure of this kind of magnetic fluid, i.e. the differential pressure it can seal is:

$$
\Delta P=\sum P_{i}=N P_{\tau}=\frac{3 N L \tau_{0}}{b},
$$

where $N$ is the number of teeth in the magnetic pole shoes.

\section{Experimental research of magnetic liquid seal}

To validate the calculation formula of magnetic liquid seal pressure, experimental research was carried out. It mainly contained the following items, the yield stress of magnetic liquid was tested firstly and then the relationship between the yield stress and seal pressure of existing magnetic liquid seal devices was analyzed.

In our research, a commercial magnetic fluid seal product was adopted, which consisted of magnetic fluid vacuum seal device and magnetic liquid. This product has been widely used in seal conditions such as vacuum, gas and so on, with its structure displayed in Fig. 3.

The performance of magnetic fluid used in this device is listed in Table 1.
Table 1

The technology guideline of magnetic fluid

\begin{tabular}{|l|c|c|c|c|}
\hline Model & CM-01 & CM-02 & CR-01 & CR-02 \\
\hline Base solution & \multicolumn{2}{|c|}{ Mineral oil } & \multicolumn{2}{|c|}{ Mineral oil } \\
\hline $\begin{array}{l}\text { Saturation magneti- } \\
\text { zation intensity, mT }\end{array}$ & 40 & 50 & 30 & 50 \\
\hline Density, kg/m 3 & 1.3 & 1.5 & 1.0 & 2.0 \\
\hline Viscosity, mPa*s & 17 & 30 & 12 & 35 \\
\hline
\end{tabular}

Yield stress testing was conducted for CM-01 and CR-01 magnetic fluid using a narrow gap magnetorheological fluid rheology property test system, with the obtained curves shown in Fig. 5.

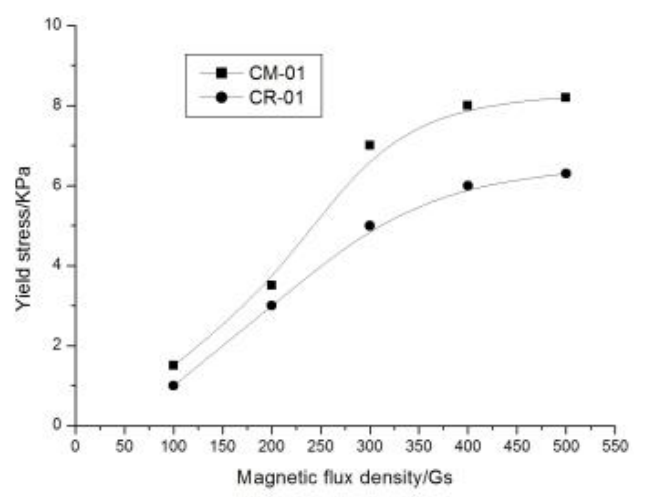

Fig. 5 The yield stress-magnetic flux density curve of magnetic fluid

The yield stress is selected from the saturated sections of above curves and calculated using Eq. (7). As calculated, $\Delta P$ is $0.1976 \mathrm{MPa}$, which is very close to $0.2 \mathrm{MPa}$ provided by the manufacturer.

\section{Conclusion}

1. In this paper, the calculation formula of magnetic liquid seal pressure is derived according to the magnetic rheology effect of magnetic liquid. And the formula is validated by testing the yield stress of magnetic liquid.

2. Determined by the rheological performance of magnetic liquid tested, the yield stress of magnetic liquid is relatively low, leading to a relatively low seal stress of magnetic liquid. Therefore, the development of magnetic liquid with high performance is a key point of the application of magnetic liquid seal.

\section{References}

1. Li, D.C.; Yang, Q.X. 2002. The study on the magnetic fluid of dryroots pump, Vacuum Science and Technology 22(4): 317-321.

2. Xing, F.F.; Li, D.C. 2011. Improvement of magnetic fluid sealing of film depositors with double shafts, Chinese Journal of Vacuum Science and Technology 31(3): 362-367.

3. Fertman, V.E. 1980 Heat dissipation in high speed magnetic fluid shaft seal, IEEE Transactions on Magnetics 16(2): 113-117. http://dx.doi.org/10.1109/TMAG.1980.1060626.

4. Zhang, J.S.; Yin, Y. S.; Zhang, S.Q. 2003. Summarization on Nanometer Magnetic Fluid and Its Application 
to Seals, Lubrication Engineering 4: 93-95.

5. Kordonsk, W.I.; Gorodkin, S.R. 1996. MRF-based Seal, Journal of Intelligent Material Systems and Structures 7: 569-572. http://dx.doi.org/10.1177/1045389X9600700518.

6. Carlson, J.D.; Jolly, M.R. 2000. Fluid, foam and elastomer device, Mechatronics 10: 555-569. http://dx.doi.org/10.1016/S0957-4158(99)00064-1.

7. Ashour, O.; Rogers, C.A.; Kordonsky, W.I. 1996. MRFs: materials, characterization, and devices, Intelligent Material Systems and Structures 7: 123-130. http://dx.doi.org/10.1177/1045389X9600700201.

8. Furst, E.M.; Gast, A.P. 2000. Micromechanics of magnetorheological suspensions, Physical Review E: Statistical Physics, Plasmas, Fluids, and Related Interdisciplinary topics 61: 6732-6739. http://dx.doi.org/10.1103/PhysRevE.61.6732.

9. Lu, S.C. 2003. Industrial Slurry-Performance, Modulation and Processing. Chemical Industry Press 5.

10. Huang, J.; Wang, H.P.; Lin, J. 2001. Research on the chain-model of the transmission mechanical property of the MRF, Machine Design and Manufacturing Engineering 30: 3-4.

11. Zhao, S.H.; Fang, J.Y. 2006. Theoretic study on the model of magnetic flocculation of magnetorheological fluid, Lubrication Engineering 11: 108-110.

12. Wang, J.X.; Meng, G. 2002. Research advances in MRFs, Acta Aeronautica et Astronautica Sinica 23: 612.

13. He, X.Z.; Li, D.C.; Hao, R.C. 2015. The influence of magnetic fluid yield stress on the performance of magnetic fluid seal, Binggong Xuebao/Acta Armamentarii 36(1): 175-181.

14. Pinho, M.; Ge'nevaux, J.M.; Dauchez, N.; Brouard, B.; Collas, P.; Me'zie're, H. 2014. Damping induced by ferrofluid seals in ironless loudspeaker, Journal of
Magnetism and Magnetic Materials 356: 125-130. http://dx.doi.org/10.1016/j.jmmm.2013.12.047.

15.Zhao, S.H.; You, F.Z. 2006. Seal Mechanism and Structure Design of Magneto-rheological Fluid, Lubrication Engineering 3: 138-139.

Sihai Zhao, Qiang Sheng, Sen Lin, Fan Zhang, Lingyu Jiao

\section{THE MODEL OF SEAL MECHANISM FOR MAGNETIC FLUID AND RELATED EXPERIMENTAL STUDY}

S u m m a r y

Magnetic fluid is a kind of magneto-rheological fluid. In this paper, the mechanism of magnetic rheology effect was proposed and the yield stress formula of magnetorheological fluid was derived, based on the dispersion and agglomeration theory of suspension liquid. In accordance with the magnetic rheology effect of magneto-rheological fluid, the computational formula of magnetic liquid seal pressure was concluded, with the seal pressure related to the yield stress of magnetic liquid and the length and clearance of leakproof structure. Using narrow gap magneto-rheological fluid rheology property test system, the yield stress of existing magnetic liquid was tested and an available seal device using magnetic liquid was studied, which proved that the calculation via formula proposed agreed well with the experimental results.

Keywords: Magnetic Fluid, Magneto-rheological Fluid, Yield Stress, Seal.

Received September 15, 2015 Accepted July 04, 2016 